\title{
ASTROMETRY USING INTERFEROMETRY AT OPTICAL WAVELENGTHS
}

\author{
K. J. JOHNSTON, D. J. HUTTER, J. A. BENSON AND \\ N. M. ELIAS II \\ US Naval Observatory, USA \\ J. T. ARMSTRONG, D. MOZURKEWICH AND T. A. PAULS \\ Naval Research Laboratory, USA \\ AND \\ C. A. HUMMEL \\ Universities Space Research Association, USA
}

\begin{abstract}
Interferometry offers an improvement in the accuracy with which astrometric measurements can be made. Using this technique, radio astronomers together with geodeticists have established a global inertial reference frame that is accurate to 0.1 milliarcseconds. At optical wavelengths, interferometry was first developed by Michelson at the turn of the twentieth century, but due to the complexities of precise beam combination at high speeds, it has lagged in its development. Now, with the availability of lasers, detectors and computers that allow path length compensation on millisecond time scales and distance determination between light collectors with a precision of $0.01 \mu \mathrm{m}$, interferometry at optical wavelengths will achieve the results in astrometry comparable to those at radio wavelengths.
\end{abstract}

\section{Introduction}

Interferometry at radio wavelengths has allowed a global inertial reference frame to be established with a precision of 0.1 milliarcseconds (mas) (Johnston et al. 1995). Relative positions of radio sources may be determined with even greater precision over small angles. After the demonstration of interferometry by Michelson \& Pease (1921) in measuring the diameters of supergiant stars such as Betelgeuse in the $1920 \mathrm{~s}$, progress was slow. These early measurements were made with a twenty foot beam attached to the 100

T.R. Bedding et al. (eds.),

Fundamental Stellar Properties: The Interaction between Observation and Theory, 39-44.

(C) 1997 IAU. Printed in the Netherlands. 
inch Hooker telescope. Later, a fifty foot interferometer was constructed on Mt. Wilson, which measured the diameters of a few more supergiant stars as well the separation of bright close binary stars such as Mizar. However, difficulties in maintaining mechanical stability and the inability to address fringe motions due to atmospheric turbulence severely limited this interferometer. In the $1960 \mathrm{~s}$, the diameters of 32 bright stars were measured by a group led by Hanbury Brown and John Davis (1974) using amplitude interferometry. In the 1970s, Shao \& Staelin (1980) demonstrated that one could recover the complex amplitude and phase of the correlated signal by using a dithered delay line. These developments led the Office of Naval Research (ONR) to initiate a program in 1982 to develop this technology for astrometry and imaging. This program resulted in the Mark III optical interferometer and the Navy Prototype Optical Interferometer (NPOI), which will be described here. The accuracy of these ground-based instruments is limited by the atmosphere. Space-based interferometers will overcome the limitations of the atmosphere, and will also be briefly described here.

\section{Ground-based Astrometry}

The principles of radio astrometry are well developed (Thompson, Moran, \& Swenson, 1986). Precise positions of stars have been determined using interferometry at radio wavelengths (Johnston et al. 1985). In these observations the Very Large Array observed the stars at several hour angles, solving for their positions from the observed phases. In Very Long Baseline Interferometry, the observed delays are used to solve for the positions. The geometry of the antennas must allow for sufficient spatial frequencies in the east-west and north-south directions to obtain an optimum solution for the celestial position. Corrections must be made for differential path delays in the instrument, atmosphere and ionosphere.

Very little has been accomplished at optical wavelengths using interferometry for astrometry. The Mark II interferometer, located on Mt. Wilson, was the first instrument capable of tracking phase and measuring fringe visibility and to report the measurement of stellar positions over large angles. The interferometer was mounted on a $5 \times 12$ foot optical table. The light collectors were siderostats with effective apertures of 3 inches, separated by $3.1 \mathrm{~m}$ along a north-south baseline. A variable delay line in the south arm was used to maintain path length equality in the arms of the interferometer. A delay line in the north arm of the interferometer induced a one-wavelength $500 \mathrm{~Hz}$ path length modulation to track and measure the parameters of the white light fringe. This allowed measurements of delay to be made over a large range of hour angles. Observations of the delay of four stars is sufficient to solve for the instrumental baseline, a constant delay 
offset, and the relative stellar positions. The $3.1 \mathrm{~m}$ baseline was measured to an accuracy of $50 \mu \mathrm{m}$ (Shao et al. 1987). This corresponds to a celestial positional accuracy of $\left(50 \times 10^{-6} \mathrm{~m} / 3.1 \mathrm{~m}\right)$ radians, or three arcseconds.

The Mark III interferometer, also located on Mt. Wilson, incorporated the developments of the Mark II. It was specifically designed to demonstrate fundamental interferometric astrometry. Star tracking and fringe tracking were completely automated to allow for rapid switching between stars. The siderostats were mounted on massive concrete piers. It had three fixed siderostat locations, giving a $12 \mathrm{~m}$ north-south baseline and a $12 \mathrm{~m}$ east-south baseline. Only one baseline could be used at a time. Light from the siderostats was directed along vacuum pipes into a temperature controlled beam combining building that contained vacuum delay lines. The stellar fringes were detected in a wide-band channel near $\lambda 0.7 \mu \mathrm{m}$ as well as narrow-band channels at 0.5 and $0.8 \mu \mathrm{m}$. Preliminary measurements with this instrument using a wide $0.3 \mu \mathrm{m}$ band centered at $0.7 \mu \mathrm{m}$ measured the $12 \mathrm{~m}$ north-south baseline to an accuracy of $6 \mu \mathrm{m}$, corresponding to a celestial accuracy of 22 mas in declination (Mozurkewich et al. 1988).

The major limitations in the accuracy of these measurements were twofold. First, turbulence in the atmosphere caused the atmospheric delay to fluctuate as $f^{-2 / 3}$, which implies that the $\mathrm{S} / \mathrm{N}$ increases as $t^{1 / 6}$, where $t$ is the integration time. By observing at two or more wavelengths, the dispersion of the atmosphere may be used to estimate the error caused by atmospheric turbulence. This two-color technique will correct for temperature microfluctuations, which are the major contribution to this error, but will not correct for turbulent water vapor fluctuations. Applying the two-color correction to all of the astrometric wide angle data obtained by the Mark III interferometer indicated an accuracy of 13 mas in declination and 23 mas in right ascension (Hummel et al. 1994). Simultaneous measurements at several wavelengths are necessary to improve the elimination of atmospheric microturbulent effects from the measurements.

The second major limitation of the Mark III was rapid variations in the baseline length (caused by imperfect manufacture of the mechanical components such as the siderostat bearings) on scales of a micron. A laser metrology system to precisely measure the positions of the siderostats relative to their massive piers was not successfully developed.

Parallel to the measurements for astrometry, a program was undertaken with the Mark III interferometer to study the spatial structure of stars. To accomplish this, a variable north-south baseline with lengths of 2 to $38 \mathrm{~m}$ was added. This instrument determined the angular diameters of over 70 stars, the orbits of 26 binary stars with separations of 3 to 120 mas, limb darkening for $\alpha$ Bootis, and the disk size of Nova Cygni, as well as other interesting astrophysical results. 
The astrometric and astrophysical results of the Mark III interferometer led to the design of a two-dimensional interferometer, the Navy Prototype Interferometer (NPOI). This instrument consists of six $50 \mathrm{~cm}$ siderostats for imaging and four $50 \mathrm{~cm}$ siderostats for astrometry. The siderostats are arranged in a $\mathrm{Y}$ shaped configuration. The astrometric siderostats are located at the center of the array and $\approx 20 \mathrm{~m}$ out along each arm. The imaging siderostats have variable spacings from 2 to $252 \mathrm{~m}$ from the center of the array along the arms of the Y. (As of January 1997, the spacings available from the center of the $\mathrm{Y}$ are 2 to $34 \mathrm{~m}$. Construction of the remaining $\mathrm{Y}$ is underway.) There are six vacuum delay lines to equalize the pathlengths between the siderostats. Vacuum delay lines are used to ensure that fringes can be simultaneously tracked and to determine the effect of atmospheric longitudinal dispersion on the fringe position. Data are taken in 32 channels distributed approximately evenly in wavenumber between $\lambda \lambda 450$ and $850 \mathrm{~nm}$. A complex metrology system consisting of over fifty lasers will measure the motions of the pivot points of the four astrometric siderostats with respect to bedrock. The expected accuracy is $0.01 \mu \mathrm{m}$.

With the array metrology system, the improved observing geometry with four simultaneous siderostats, and the extension of the two-color dispersion correction method to use several of the 32 spectral channels, it is expected that the baselines will be measured to an accuracy of $0.1 \mu \mathrm{m}$, resulting in an accuracy in celestial position, with a $20 \mathrm{~m}$ baseline, of 1 mas.

All of the previous discussion deals with measurements over wide angles, for which the astrometric accuracy $\sigma_{\theta}$ improves as $t^{-1 / 6}$. For narrow-angle astrometry (separations smaller than $\sim 10^{\prime}$ ), $\sigma_{\theta} \propto t^{-1 / 2}$ (Lindegren 1980). Narrow-angle astrometry is itself divided into two regimes. For $\theta<B / h$, where $h$ is the height of the dominant atmospheric turbulence, $\theta$ is the angle between the positions of the two stars, and $B$ is the baseline length, $\sigma_{\theta} \propto$ $B^{-2 / 3} \theta t^{-1 / 2}$. For the $\theta>B / h$ case, $\sigma_{\theta} \propto \theta^{1 / 3} t^{-1 / 2}$. These dependencies have been verified by Han (1991) for the $\theta>B / h$ case and Colavita (1994) for the $\theta<B / h$ case, for which the error is consistent with $21 \mu$ as $/ \sqrt{t}$ for a $12 \mathrm{~m}$ baseline with the Mark III interferometer. The Palomar Testbed Interferometer (PTI) now under fabrication by JPL is specifically designed for the $\theta<B / h$ case in that it can simultaneously observe two very closely separated stars with a long baseline. This system operates at $\lambda 2 \mu \mathrm{m}$ and should come on line in 1998.

\section{Space Astrometry}

The advantage of space interferometry for astrometry can be clearly seen from the previous discussion. Absence of the atmosphere means that the astrometric accuracy is limited only by the knowledge of the interferome- 
ter geometry. In addition, larger apertures can be used so that very faint objects can be observed. There have been many systems proposed. The two most prominent systems in the early 1990s were POINTS, proposed by SAO, and OSI, proposed by JPL. The leading contender is now the Space Interferometry Mission (SIM), an offshoot of OSI, that will be capable of $4 \mu$ as accuracy. This instrument consists of a linear array of seven apertures with spacings from 1 to $10 \mathrm{~m}$ making up three interferometers, two for pointing and one for scientific data. Expected launch is 2004 or later.

\section{Future Prospects}

Future prospects look excellent for both ground and space based astrometric interferometers. The previous discussion has shown that very little has been accomplished thus far. The instruments are very complex, and a large amount of resources is needed to develop them. The author list on this paper gives testament to these facts. The ONR support in this area has been crucial in developing the technology in this area. As a result of this support, the NPOI is nearing completion. NASA, through its interest in extra-solar planets, is developing the PTI, which also is nearing completion, and is also beginning to seriously support a space based mission, SIM.

The NPOI as of January 1997 is very near completion. Three of the astrometric siderostats, one in the center of the array and those on the east and west arm, are in operation nightly. The system is being debugged for automated operation in order to achieve the large number of observations needed for astrometry, say 200 per night. At this time, about 100 observations per night have been realized. The metrology system for the astrometric siderostats is nearing completion. The fourth siderostat is expected to be in operation by the fall of 1997 . With this, the instrument will have full capability for astrometric observations.

The NPOI has achieved some significant milestones. Using simultaneous multichannel closure phase and visibility amplitude data, it has imaged the spectroscopic binary star Mizar A (Benson et al. 1997) with three siderostats and baselines of 19,22 , and $38 \mathrm{~m}$. These are the first phase closure $\left(\phi_{\mathrm{C}}\right)$ and squared visibility amplitude $\left(V^{2}\right)$ measurements made simultaneously in multiple spectral channels. Figures 1c and 1d of Hummel $\&$ Benson (1997 [this volume]) display $V^{2}$ and $\phi_{\mathrm{C}}$ versus wavelength for an observation obtained on 1996 May 1 . The closure phases show a $180^{\circ}$ phase jump at the minimum in $V^{2}$, confirming earlier results that show that the components of Mizar A are almost identical (Hummel et al. 1995). Data from several scans on 1996 May 1 were processed using the standard techniques of radio astronomy to form an image of Mizar A, displayed in Fig. 1a of Hummel \& Benson (1997). This image has a dynamic range of 
approximately 100:1 and a spatial resolution of 3 mas.

Another significant result in obtaining precise values for stellar parameters has also been demonstrated. Multichannel observations of $\alpha$ Cas have been obtained at several hour angles. Figure 2 of Hummel \& Benson (1997) displays the amplitude of the triple product (the product of the complex visibilities on the three baselines). These data allow a precise model for the stellar size and limb darkening to be made. For $\alpha$ Cas, Hajian et al. (1997) have determined a linear limb-darkening coefficient of 0.4 and an angular diameter of 5.58 mas, with a formal error of $5 \mu$ as. This result demonstrates the capability of optical interferometry to determine stellar diameters at the $0.1 \%$ level.

These two results clearly demonstrate that optical interferometry has matured, substantially overcoming many of the technical problems of operating at optical wavelengths. By the year 2000 , the ground-based systems will produce significant results. The addition of space-based instruments in the $21^{\text {st }}$ century will further these results, especially in giving sub-milliarcsecond positions over wide angles.

\section{References}

Benson, J. A., Hutter, D. J., Elias, N. M. II, Bowers, P. F., Johnston, K. J., Hajian, A. R., Armstrong, J. T., Mozurkewich, D., Pauls, T. A., Rickard, L. J, Hummel, C. A., White, N. M., Black, D., \& Denison, C. S. 1997, AJ, submitted

Brown, R. H., Davis, J., \& Allen, R. L. 1974, MNRAS, 167, 121

Colavita, M. M. 1994 A\&A 283, 1027

Hajian, A. R., et al. 1997, to be submitted to ApJ

Han, I. 1989, AJ, 97, 607

Hummel, C. A., Armstrong, J. T., Buscher, D. F., Mozurkewich, D., Quirrenbach, A., \& Vivekanand, M. 1995, AJ, 119, 376

Hummel, C. A., \& Benson, J. A. 1997, this volume

Hummel, C. A., Mozurkewich, D., Elias, N. M. II, Quirrenbach, A., Buscher, D. F., Armstrong, J. T., Johnston, K. J., Simon, R. S. \& Hutter, D. J. 1994, AJ, 108, 326

Johnston, K. J., deVegt, C., Florkowski, D. M., \& Wade, C. M. 1985, AJ, 90, 2390

Johnston, K. J., Fey, A. L., Zacharias, N., Russell, J. L., Ma, C., deVegt, C., Reynolds, J. L., Jauncey, D. L., Archinal, B. A., Carter, M. S., Corbin, T. E., Eubanks, T. M., Florkowski, D. M., Hall, D. M., McCarthy, D. D., McCulloch, D. M., King, E. A., Nicholson, G., \& Shaffer, D. B. 1995, AJ, 110, 880

Lindegren, L. 1980, A\&A, 89, 41

Michelson, A. A. \& Pease, F. G. 1921, ApJ, 53, 249

Mozurkewich, D., Hutter, D. J., Johnston, K. J., Simon, R. S., Shao, M., Colavita, M. M., Staelin, D. H., Hines, B., Hershey, J. L., Hughes, J. A., \& Kaplan, G. H. 1988, AJ, 95, 1296

Shao, M. \& Staelin, D. H. 1980, J. Opt. Soc. Am., 19, 1519

Shao, M., Colavita, M. M., Staelin, D. H., Johnston, K. J., Simon, R. S., Hughes, J. A. \& Hershey, J. L. 1987, AJ, 93, 1280

Thompson, A. R., Moran, J. M. \& Swenson, G. W. 1986, Interferometry and Synthesis in Radio Astronomy (Wiley, New York) 